\title{
Reproductive system of the genus Crasiella (Gastrotricha, Macrodasyida)
}

\author{
Loretta Guidi • M. Antonio Todaro • \\ Marco Ferraguti $\cdot$ Maria Balsamo
}

Received: 27 April 2010/Revised: 1 July 2010/ Accepted: 2 July 2010/Published online: 13 July 2010

(C) Springer-Verlag and AWI 2010

\begin{abstract}
Crasiella diplura from Sweden and Crasiella sp. from Italy were studied alive and with transmission electron microscopy (TEM). The two species are simultaneous hermaphrodites and share the same reproductive system lay-out: paired ovaries extend along the posterior part of the intestine and join mid-dorsally, while bilateral, club-shaped testes lie at the sides of the anterior gut, extending as deferentia that fuse on the mid-ventral plane and open into a single pore; gametes mature in a caudocephalic and centripetal direction. The bulky, sac-like, frontal organ is lined by a simple epithelium and lies dorsolaterally to the intestine, on the left side of the body. The spindle-shaped caudal organ is musculo-glandular and is located ventrolaterally to the gut on the right side. It is characterized by the presence of a roughly Y-shaped internal channel that opens into two pores close to each other, which function for the intake and outlet of the (auto)sperm, respectively. The spermatozoa, which are peculiar and similar in the two species, are characterized by a long and complex acrosome consisting of four ultrastructurally distinct regions, three of which find
\end{abstract}

Communicated by H.-D. Franke.

L. Guidi $(\bowtie) \cdot$ M. Balsamo

Dipartimento di Scienze dell'Uomo,

dell'Ambiente e della Natura,

Università di Urbino 'Carlo Bo', Urbino, Italy

e-mail: loretta.guidi@uniurb.it

\section{A. Todaro}

Dipartimento di Biologia,

Università di Modena-Reggio E, Modena, Italy

M. Ferraguti

Dipartimento di Biologia, Università di Milano,

Via Celoria 26, 20133 Milan, Italy equivalence in other gastrotrich species. The flagellum lacks a striated cylinder. Anatomy and ultrastructure enable us to hypothesize a modality of sperm transfer in Crasiella that is similar to that observed in Macrodasys.

Keywords Crasiella $\cdot$ Gastrotricha $\cdot$ Macrodasyida . Reproductive system $\cdot$ Spermatozoon

\section{Introduction}

Gastrotricha is a phylum comprising about 750 species of acoelomate microinvertebrates, which are well represented in benthic communities and are grouped into two orders: Macrodasyida, consisting of 300 marine species, which are generally simultaneous or sequential hermaphrodites with cross-fertilization, and Chaetonotida, comprising 450 mostly freshwater, parthenogenic species, with few marine, hermaphroditic genera (Neodasys, Heteroxenotrichula, Xenotrichula, Diuronotus, Musellifer) (Balsamo et al. 2009; Hummon and Todaro 2010).

The reproductive system of Macrodasyida is anatomically quite complex and diverse, probably because of the different modalities of internal cross-fertilization. Usually, there is a pair of testes, two (or one) ovaries and two sexual accessory organs, which are respectively known as frontal and caudal organs from their relative positions in the trunk region (Ruppert 1978a). The elongated testes lie at the sides of the anterior intestine and extend posteriorly in sperm ducts, opening ventrally into either a common or separated pores. The ovaries are lateral to the caudal intestine, and oocytes generally mature in a caudo-frontal direction, gradually shifting dorsally. In some taxa (e.g. Cephalodasys and Dolichodasys), oocytes mature in a fronto-caudal direction. The dorsal fusion of the two 
ovaries in order to form an unpaired uterus has been reported in Dactylopodola typhle (Kieneke et al. 2008). A connection made of either a solid cell band or an epithelial duct between the ovary and an external, dorsal pore (mating pore?), has only been described in Acanthodasys thrinax and Diplodasys ankeli (Thaumastodermatidae), and Dactylopodola typhle, respectively (Ruppert 1978b; Kieneke et al. 2008).

The sac-like frontal organ is lined by an epithelium and lies in proximity to the most mature egg; it displays an external pore through which it probably receives allosperm during copulation, thus functioning as a seminal receptacle. An internal pore allows the stored sperm to leave the organ and fertilize the eggs. The elongated caudal organ is wholly muscularized and lies ventrally; it opens close to or within the anus and almost certainly acts as a copulatory organ (Ruppert 1991). In the Macrodasys species, the caudal organ forms a thin protrusile tube within which the autosperm are packed (Ruppert 1978a), while in some Urodasys species, it has a cuticularized stylet (Schoepfer-Sterrer 1974; Fregni et al. 1998; Balsamo et al. 2007). Mating and sperm cross-transfer modalities have been described in detail by Ruppert (1978a, b) on the basis of ultrastructural observations of Macrodasys sp. and different species of Thaumastodermatidae. In addition, hypotheses about these processes have been advanced by Teuchert (1968), Balsamo et al. (2002), Kieneke et al. (2008) and Guidi et al. (2009) based upon microscopical and ultrastructural data on species of other families.

The studies carried out in the last 30 years have proved that the morphology of the reproductive organs and the ultrastructure of spermatozoa are very important diagnostic characters for taxonomic use, as well as being a valid tool for phylogenetic purposes (e.g. Thane-Fenchel 1970; Evans 1994; Marotta et al. 2005; Kieneke et al. 2009).

However, no morphological data on the reproductive organs and spermatozoa of the Planodasyidae family are yet available. Herein, we provide a description of the reproductive system and the spermatozoon of two species of Crasiella, which is one of the two genera of the family. We also suggest a possible modality of mating and fertilization in the two studied species of this genus and compare this to the information available on other species of Macrodasyida.

\section{Methods}

Sand samples of Crasiella diplura Clausen, 1968 were collected in September 2007 during a 2-week workshop held at the Sven Lovén Centre for Marine Sciences on Tjärnö, an island on the Swedish west coast (for details see Willems et al. 2009). Sand samples of Crasiella sp. had also been collected in July 2002 at S. Isidoro, Lecce, Italy. Gastrotrichs were extracted from the sediment by the narcotization-decantation technique, using an isosmotic (7\%) magnesium chloride solution (Giere 2009). The faunacontaining supernatant was then poured directly into a 5-cm diameter Petri dish and scanned for specimens under a Wild M3 dissecting microscope set at 50× magnification. For optical microscopy, the gastrotrichs were removed with a micropipette from the Petri dish, fresh-mounted on slides and observed using a Nikon Eclipse 80i equipped with Differential Interference Contrasts (Nomarski). During observation, the animals were photographed with a DS$5 \mathrm{M}$ Nikon digital camera and measured with the Nikon NIS software.

For the transmission electron microscopy, 10 specimens of Crasiella diplura and five of Crasiella sp. were fixed overnight in $2 \%$ glutaraldehyde in a $0.1 \mathrm{M}$ sodium cacodylate buffer ( $\mathrm{pH}$ 7.4) and stored in a $0.1 \mathrm{M}$ sucrose/ sodium cacodylate buffer. In the laboratory, the specimens were post-fixed in $1 \%$ osmium tetroxide buffered with $0.1 \mathrm{M}$ sodium cacodylate, washed in a clean $0.1 \mathrm{M}$ sodium cacodylate buffer, dehydrated in a graded ethanol series and embedded in Araldite. Semi-thin and ultra-thin sections were cut with a LKB Ultrotome $2088 \mathrm{~V}$ and were contrasted with toluidine blue and lead citrate, respectively. The semi-thin sections were observed in transmission light under a VANOX AHBT3 Olympus optical microscope, whereas the ultra-thin sections were observed under a Philips CM10 transmission electron microscope. The locations of some of the morphological characteristics along the body are given in percentages of units $(\mathrm{U})$ of the total body length, measured from the anterior to the posterior.

Three-dimensional reconstruction of the spermatozoon of $C$. diplura was performed by CimatronE software (Cimatron Ltm, Tel Aviv, Israel; www.cimatrom.com).

\section{Results}

Reproductive apparatus

The overall arrangement of the reproductive system is the same in the two species of Crasiella examined. Mature individuals are simultaneous hermaphrodites. One pair of club-shaped testes lies at the sides of the anterior gut, extending into two sperm ducts that fuse on the mid-ventral plane and open into a single ventral pore (Fig. 1a-c, e). Two bilateral ovaries run along the posterior part of the intestine and join mid-dorsally (Fig. 1a, d). In the posterior trunk region, there are two accessory organs, both of which are very close to each other. The voluminous, sac-like frontal organ is lined by an epithelium and lies 

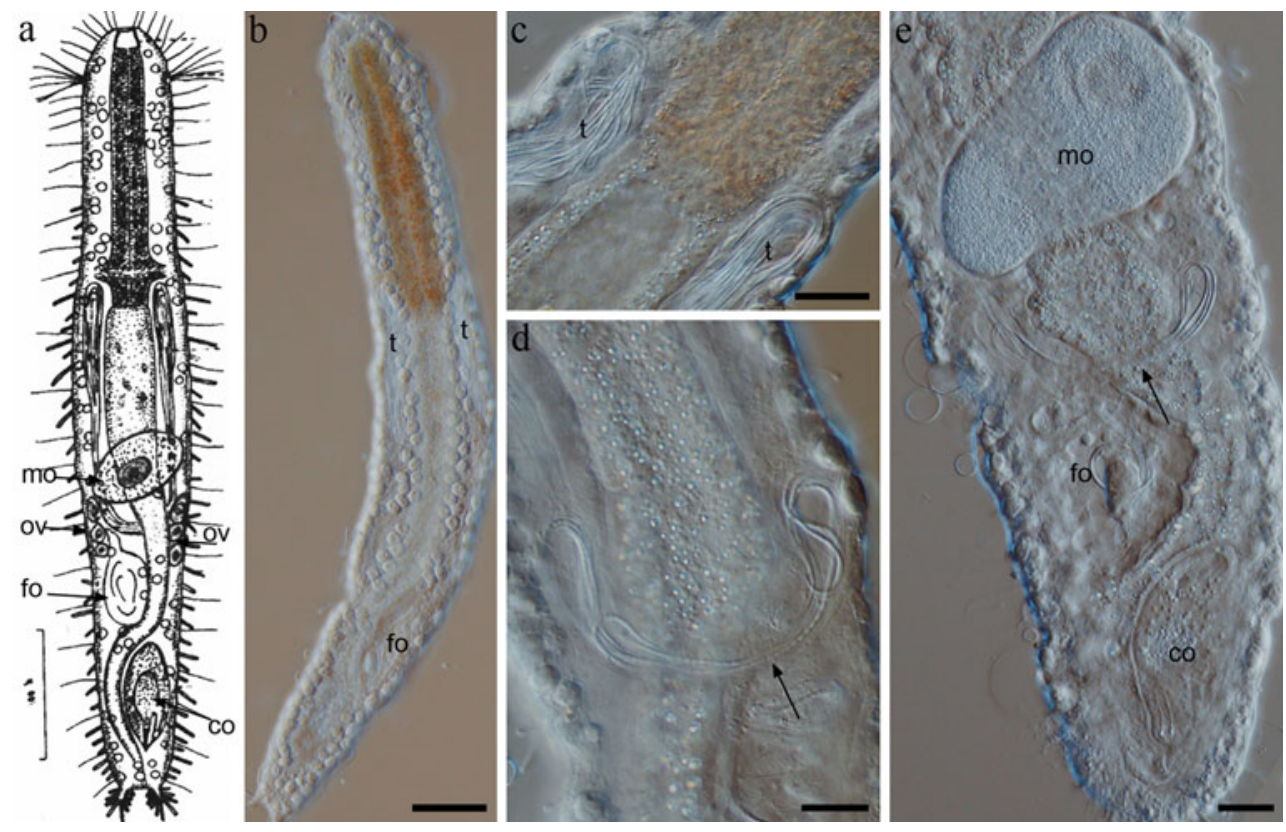

Fig. 1 Morphology of the reproductive system of Crasiella diplura (b, c, d, e DIC microscopy). a Drawing of a dorsal view of Crasiella diplura showing the reproductive system (modified from Clausen 1968). b Habitus of an adult specimen c Proximal portion of the testes at the level of the pharingeo-intestinal junction. d Fusion of the sperm

dorsolaterally to the intestine on the left side of the body, between the largest oocyte and the caudal organ. The spindle-shaped caudal organ is musculo-glandular and located ventrolaterally to the gut on the right side of the body (Fig. 1a, d).

\section{Testes}

The two testes extend from U34 to U50 and narrow into sperm ducts, which open into a single ventral pore at U66. The germinal epithelium is arranged along the entire length of each testis, and gametes mature in a caudo-cephalic and centripetal direction so that early germinal cells, spermatogonia and spermatocytes lie in the posterior part of each gonad, while spermatids gather in the internal lumen of the most anterior part (Fig. 2a).

\section{Spermatogenesis}

In spermatids, the nuclear chromatin progressively condenses, while the nucleus starts elongating. At the same time, a long mitochondrion, arising from the fusion of many smaller mitochondria, sticks to the nuclear envelope and gradually surrounds it, before then sinking into the nucleus. A manchette of microtubules is visible around the nucleus-mitochondrion complex (Fig. 2a, b). At that point, the acrosome starts to develop: the cytoplasm of the spermatid is almost entirely filled with large vesicles containing ducts on the mid-ventral plan (arrow). e Posterior region of the body: the mature oocyte, the fusion of the sperm ducts (arrow), and the frontal and caudal organs are clearly visible. co caudal organ, fo frontal organ, mo oocyte, ov ovary, $t$ testes. Scale bars $\mathbf{b}=50 \mu \mathrm{m}$; $\mathbf{c}-\mathbf{e}=15 \mu \mathrm{m}$

a homogeneous, moderately electron-dense material, and numerous small, strongly electron-dense vesicles. These progressively pile up, forming the basal portion of the acrosome, while their content condenses. As the nuclearacrosomal complex is growing, the axoneme is already apparent and starts lengthening (Fig. 2c, d). Once the nucleus has reached its definitive size, it rotates medially and then posteriorly with respect to the axoneme. Due to this transformation, the spermatid takes on a clear U-shape (Fig. 2e). When the acrosome formation has been completed, spermatids enter the lumen of the anterior portion of each testis and move in a caudal direction. Their flagellum rotates with respect to the nucleus, getting in line with the nuclear-acrosomal complex. Mature spermatozoa migrate caudally along the testis lumen towards the sperm duct, which is completely surrounded by circular muscles belonging to the trunk musculature (Fig. 2f).

\section{Spermatozoa}

The spermatozoon of Crasiella diplura is long, filiform cells containing, in sequence, an acrosome, a nucleusmitochondrial complex and a tail. The acrosome is very long (about $50 \mu \mathrm{m}$ ) and is subdivided into four different morphological regions over its length (Figs. $2 \mathrm{~g}, 3 \mathrm{a}, \mathrm{b}$ ). The apical region, which is $8 \mu \mathrm{m}$ long, is a helically twisted tube with an inner structure made of piled discs (Fig. 2h, o); its diameter increases in a basal direction 

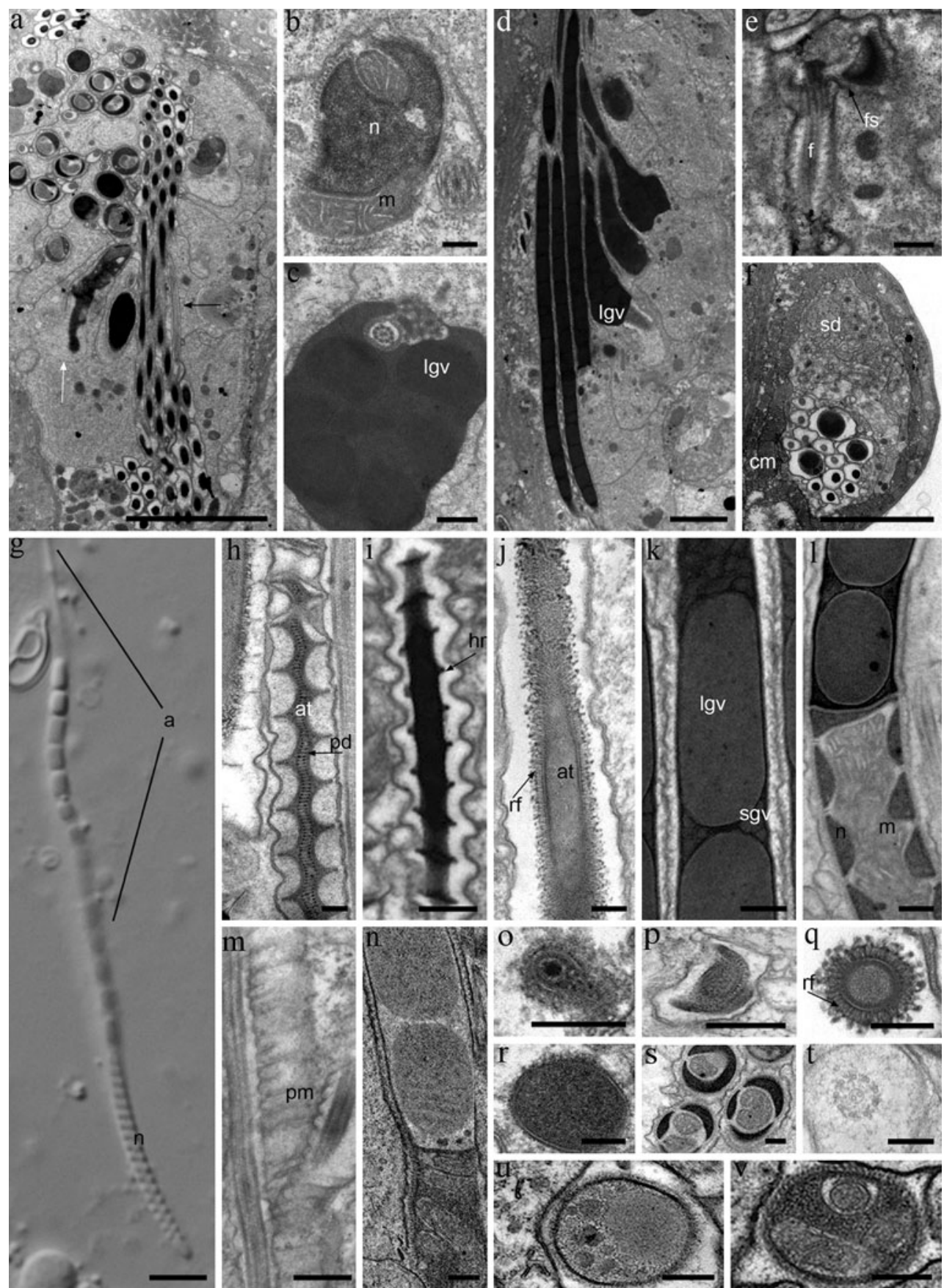

from 0.1 to $0.2 \mu \mathrm{m}$. The next region is a straight tube, which is $7 \mu \mathrm{m}$ in length and surrounded by a helical ridge (Fig. 2i, p), with a diameter increasing from 0.2 to $0.3 \mu \mathrm{m}$. The third region, $4 \mu \mathrm{m}$ long, is a straight tube surrounded by numerous short radial fibres (Fig. 2j, q). The last region is much larger, being $31 \mu \mathrm{m}$ in length and $0.8 \mu \mathrm{m}$ in diameter, and has large, piled, globular vesicles $(0.7 \mu \mathrm{m}$ in diameter). Between these large vesicles, there are several smaller globular vesicles $(0.14 \mu \mathrm{m}$ in diameter), which are embedded within a very electron-dense material (Fig. 2k, r).
The nucleo-mitochondrial portion is about $20 \mu \mathrm{m}$ long and has a mitochondrion $(0.5 \mu \mathrm{m}$ in diameter $)$ that is surrounded by the nucleus, forming a characteristic "spring", which is a ribbon of compacted chromatin spirally coiled around the mitochondrion for a total of 20 turns (Figs. 2g, 1, s, 3a, b). The flagellum has a $9 \times 2+2$ axoneme, characterized by a dense and prominent central sheath surrounding the central tubules; a wide space separates the axoneme from the tail membrane. Another peculiarity of the sperm tail in Crasiella is the fact that, apparently, the plasma membrane is curled (Figs. 2m, t, 3a, b). 
4 Fig. 2 Spermatogenesis of Crasiella diplura and the spermatozoa of C. diplura and Crasiella sp. (All figures refer to $C$. diplura except $\mathbf{n}$, $\mathbf{u}, \mathbf{v} ; \mathbf{a}-\mathbf{f}, \mathbf{h}-\mathbf{v}$ are TEM images, $\mathbf{g}$ is a DIC image). a Testes: the gametes mature in a caudo-cephalic (white arrow) and centripetal direction (black arrow). b Nucleus/mitochondrion of a spermatid: the chromatin starts to condense and the mitochondrion is surrounding the nuclear envelope. c Spermatid showing the cytoplasm filled with large vesicles from which the basal region of the acrosome develops. d Basal regions of acrosomes: the large vesicles are piled up on top of each other. e The U-shaped connection between nucleus and flagellum in a late spermatid. f Sperm duct in longitudinal section. g A single spermatozoon as seen by differential interference contrast optics. $\mathbf{h}$ The more apical region of the acrosome of in longitudinal section: a twisted tube with an inner structure made up of piled discs. i The second region of the acrosome of in longitudinal section: a straight tube surrounded by a helical ridge. $\mathbf{j}$ The third region of the acrosome in longitudinal section: note the small radial fibres visible also in cross-section in q. $\mathbf{k}$ The more proximal region of the acrosome in longitudinal section formed by large, piled, globular vesicles surrounded by smaller globular vesicles. I Longitudinal section of the nuclear-mitochondrial region and the basal acrosome. m Tangential section of the curled plasma membrane of the flagellum. n Longitudinal section of connection between the acrosome and nuclear-mitochondrial region of Crasiella sp.: note the thin mitochondrion and the low and the high density of large and small globular vesicles, respectively. Note also the low density of the granular material surrounding both types of vesicles. o-r Crosssections of the acrosome, ordered from distal to proximal. s Crosssection of nuclear-mitochondrial region. $\mathbf{t}$ Cross-section of the flagellum: note the prominent central sheath. $\mathbf{u , v}$ Cross-section of the proximal region of the acrosome and the nuclear-mitochondrial region of Crasiella sp. a acrosome, at acrosomal tube, $\mathrm{cm}$ circular muscles, $f$ flagellum, $f s$ final spermatid, $h r$ helical ridge, $l g v$ globular vesicles, $m$ mitochondrion, $n$ nucleus, $p m$ plasma membrane, $p d$ piled discs, $r f$ radial fibres, $s d$ sperm duct, $s g v$ small globular vesicles. Scale bars $\mathbf{a}=4 \mu \mathrm{m} ; \mathbf{b}, \mathbf{d}, \mathbf{e}, \mathbf{h}, \mathbf{i}-\mathbf{s}, \mathbf{u}, \mathbf{v}=0.3 \mu \mathrm{m} ; \mathbf{c}, \mathbf{f}, \mathbf{g}=4 \mu \mathrm{m}$; $\mathbf{t}=0.1 \mu \mathrm{m}$

The spermatozoon of Crasiella sp. differs from that of Crasiella diplura in the following ways: (1) the large globular vesicles of the acrosomal base are less electrondense; (2) the small globular vesicles measure 0.07 versus $0.14 \mu \mathrm{m}$ in diameter and are more electron-dense; (3) the granular material surrounding the vesicles is less electrondense; (4) the mitochondrion inside the nucleus is much thinner, 0.18 versus $0.5 \mu \mathrm{m}$ (Fig. $2 \mathrm{n}, \mathrm{u}, \mathrm{v}$ ).

\section{Ovaries}

The two lateral ovaries extend along the intestine from U60 to U76. Each ovary is formed by a few oocytes (4-5) that migrate anteriorly and dorsally during maturation. The fully grown oocyte lies anterior to the frontal organ and dorsal to the intestine (Fig. 4a-d). No epithelial sheath enveloping the germ cells was observed. Circular and longitudinal trunk muscles surround the ovaries. The early oocytes have many free ribosomes and mitochondria; the nuclear chromatin is granulated and moderately electrondense, and the nucleolus is large and well organized (Fig. 4b). Later oocytes contain yolk granules, which
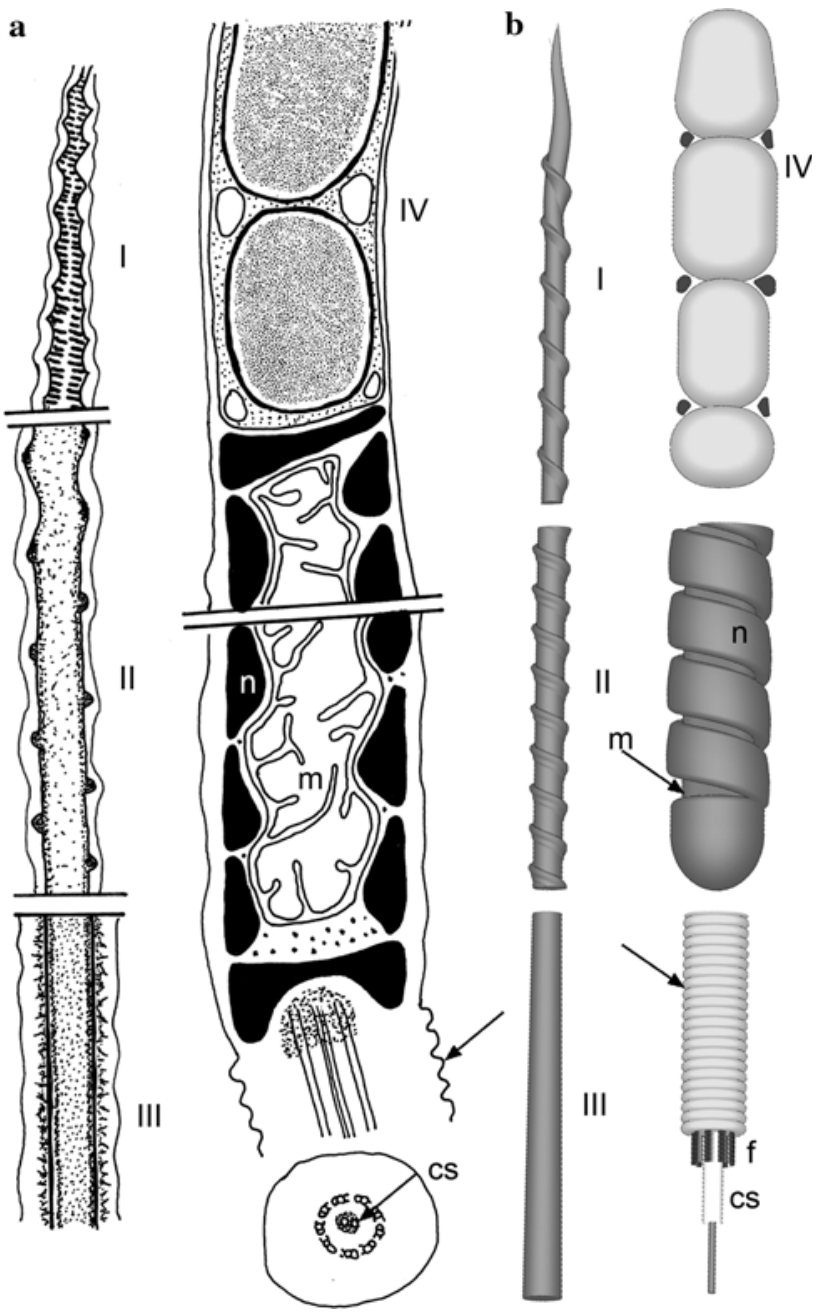

Fig. 3 Graphic reconstructions of the mature spermatozoon of Crasiella diplura. a Schematic drawing of the spermatozoon. I-IV: different regions of the acrosome, from the more distal (I) to the more proximal (IV) (see the description in the text). The nucleus is spiralized around the mitochondrion and the flagellum is surrounded by a curled plasma membrane (arrow). b 3D model of the spermatozoon showing the same regions of the spermatozoon represented in the $2 \mathrm{D}$ drawing. cs central sheath; $f$ flagellum(* in cross-section); $m$ mitochondrion; $n$ nucleus

progressively increase both in number and size during oogenesis, until they finally fill the entire cytoplasm. The nuclear chromatin is moderately electron-dense, and the nucleolus is absent (Fig. 4c). Mature oocytes are very large cells $(100 \mu \mathrm{m}$ in diameter), which are packed with yolk granules, free ribosomes and fragments of rER. The egg envelope consists of a thin, inner layer of electron-dense granular material and an outer layer of densely packed microfilaments (Fig. 4d, e).

\section{Frontal organ}

The frontal organ is a large sac ( $86 \mu \mathrm{m}$ long, $26 \mu \mathrm{m}$ wide), extending from U65 to U80. An external, well-defined 

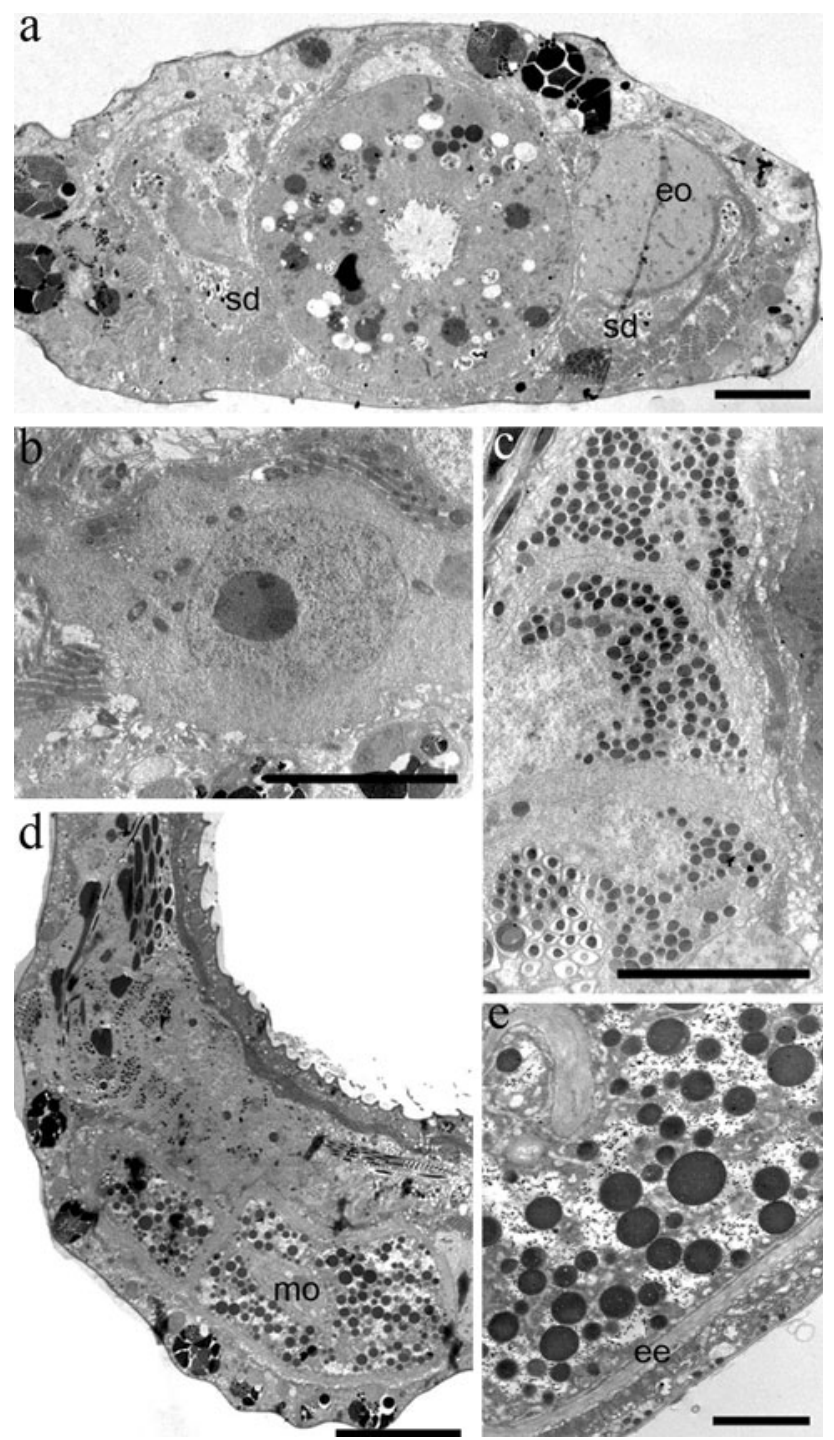

Fig. 4 Ovaries of Crasiella diplura (TEM microscopy). a Crosssection of the body of a whole animal at the level of the early oocytes. The sperm ducts are sectioned close to their fusion. b An early oocyte. c Three later oocytes. d Mature dorsal oocyte packed with yolk granules (longitudinal section). e Egg envelope: the inner thin layer made of electron-dense granular material and the outer one made of densely packed microfilaments are visible. $e e$ egg envelope, $e o$ early oocyte, mo mature oocyte, $s d$ sperm duct. Scale bars $\mathbf{a}-\mathbf{d}=8 \mu \mathrm{m}$; $\mathbf{e}=1 \mu \mathrm{m}$

dorsolateral pore is present, and another small pore is directed anteriorly towards the most mature oocyte. The frontal organ is lined by a monolayered columnar epithelium, delimiting a cavity in which usually numerous active spermatozoa are visible in living specimens (Fig. 5a). The epithelial cells have many microvilli extending into the inner cavity, and a cytoplasm with secretory vesicles of different sizes, varying from 0.2 to $1.5 \mu \mathrm{m}$. The spermatozoa inside the lumen are surrounded by a uniformly electron-dense material. Circular and longitudinal muscles, belonging to the trunk musculature, surround the frontal organ (Fig. 5c).

\section{Caudal organ}

The caudal organ is spindle-shaped (70 $\mu \mathrm{m}$ long, $30 \mu \mathrm{m}$ large) and extends from U73 to U84. It is formed by an anterior, glandulo-muscular region $(50 \mu \mathrm{m}$ long) and by a posterior muscular region ( $20 \mu \mathrm{m}$ in length). This organ is completely surrounded by a thick basal lamina and welldeveloped circular muscles and also contains an internal duct (Figs. 5b, f, 6).

This internal duct is Y-shaped, with one branch lying inside the anterior region and forming a blind end (Figs. 5b, e, 6), while the other two branches extend along the posterior region and open into the exterior through two distinct ventral pores (Figs. 5b, e, f, g, 6).

The anterior, blind duct is surrounded by cells with the cytoplasm full of secretory granules. Numerous microvilli extend into the lumen. Inside it, in some cases, spermatozoa were observed (Fig. 5d, e).

Of the two ducts lying in the posterior region, the main duct is $10 \mu \mathrm{m}$ long and $8 \mu \mathrm{m}$ wide and ends with a 'feeder bib' extremity (12 $\mu \mathrm{m}$ long), which opens into a very small ventral pore $(1.5 \mu \mathrm{m}$ in diameter, Figs. $5 \mathrm{~b}, 6)$. The second duct, the accessory one, is straight and much longer $(25 \mu \mathrm{m}$ long, $3 \mu \mathrm{m}$ wide). It lies ventrally to the first and opens into a large ventral pore $(6 \mu \mathrm{m}$ in diameter) (Figs. 5f, 6). The lumen of the main duct is covered with a large number of packed secretion granules, which are small, round or ovoid in shape, and contains a homogeneous, moderately electron-dense material. The cytoplasm of the cells surrounding the lumen is filled with secretion granules $(80 \times 200 \mathrm{~nm})$, globular mitochondria, numerous stacked membranes (endoplasmic reticulum and dictyosomes), ribosomes that are both free and attached to the reticulum membranes, and many groups of secretion granules. The nuclei often have annulated membranes (Fig. 5e, j). These cells also form many, very long and extremely thin cytoplasmatic protrusions, which overlap each other, thus forming a multimembranous sheath all over the lumen. The secretion granules are regularly stuck into these cytoplasmic protrusions. The content of the granules is progressively released into the lumen and appears as a granular material (Fig. 5e, k). The lumen of the feeder bib-shaped extremity of the main duct is covered with typical secretion granules, which are ovoid in section, slightly electron-dense, and are regularly arranged in rows. Only the terminal part of the duct lacks these granules and is wholly surrounded by large cells that are peculiar for their irregular shape and numerous interdigitations. They contain many mitochondria, a well-developed Golgi apparatus, a lot of ribosomes 

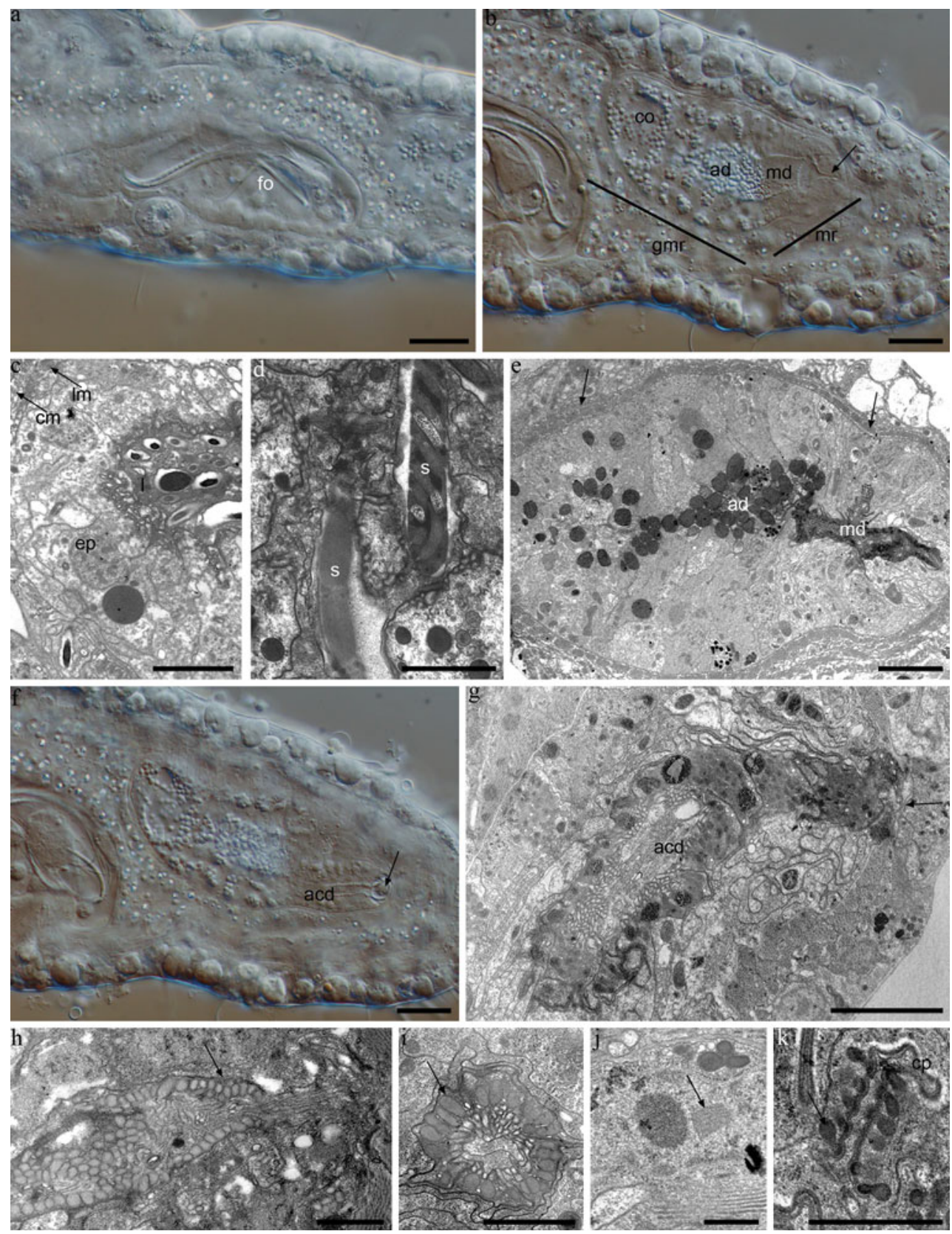

Fig. 5 Accessory organs of Crasiella diplura (All figures refer to C. diplura except $\mathbf{d} ; \mathbf{c}-\mathbf{e}, \mathbf{g}, \mathbf{h}$ are TEM images, $\mathbf{a}, \mathbf{b}, \mathbf{f}$ are DIC images). a The frontal organ containing several spermatozoa. b The caudal organ. The anterior, blind duct surrounded by secretory cells and the posterior, main duct ending in the 'feeder bib' extremity (arrow) are clearly visible. c Epithelium of the frontal organ, which surrounds the lumen containing spermatozoa. d Spermatozoa inside the anterior blind duct of the caudal organ in Crasiella sp. e Caudal organ showing the well-developed circular muscles (arrows), the anterior blind duct and the posterior main duct. f Caudal organ in which the accessory duct opening into a large pore (arrow) is visible. g Accessory posterior duct of the caudal organ. The pore (arrow) is visible. h, i Longitudinal (h) and cross-sections (i) of the 'feeder bib' extremity of the principal, posterior duct. Note the arrangement of its typical secretion granules (arrows). $\mathbf{j}$ Annulated membranes (arrow) in the nucleus of the cells surrounding the principal, posterior duct. $\mathbf{k}$ The cytoplasmatic protrusions forming a multi-membranous sheath all over the lumen of the main duct. Note the regular arrangement of the secretion granules (arrow) inside these cytoplasmic protrusions. acd accessory duct, ad anterior blind duct, $\mathrm{cm}$ circular muscles, $c o$ caudal organ, $c p$ cytoplasmic protrusion, $e p$ epithelium of frontal organ, fo frontal organ, $g m r$ glandulo-muscular region, $l$ lumen, Im longitudinal muscles, $m d$ main duct, $m r$ muscular region, $s$ spermatozoon. Scale bars $\mathbf{a}, \quad \mathbf{b}, \mathbf{f}=15 \mu \mathrm{m} ; \mathbf{c}, \quad \mathbf{d}=2 \mu \mathrm{m}$; $\mathbf{e}, \mathbf{g}=8 \mu \mathrm{m} ; \mathbf{h}-\mathbf{k}=1 \mu \mathrm{m}$ 
that are both free and attached to the rER membranes and secretion granules (Fig. 5h, i).

The accessory duct is connected to the anterior end of the main duct and extends posteriorly along the medial side of the caudal organ up to a ventral pore that is clearly visible in both light and electron microscopy (Fig. 5f, g). The lumen of the accessory duct is covered with at least three types of secretion granules: the first kind are large and ring-shaped and made up of coarse granular material; the second kind are ovoid or round and full of coarse granular material; the third kind are small, cylindrical granules and consist of homogeneous material. The cells surrounding the lumen have many organelles and groups of all of the types of secretion granules. Numerous elongated microvilli extend from the cell surface into the luminal space (Fig. $5 \mathrm{~g}$ ).

\section{Discussion}

Members of the Crasiella genus are hermaphroditic, with separate female and male gonads, as is the case in most species of Macrodasyida. The paired condition of both testes and ovaries is very common in macrodasyidan gastrotrichs, as is the caudo-cephalic direction of the maturation of spermatozoa and oocytes (Ruppert 1991; Fischer 1996; Balsamo et al. 2002).

The presence in Crasiella of two club-shaped and hollow testes, which extend into sperm ducts confirms the basic plan of the male system of Macrodasyida (Ruppert 1991; Fischer 1994; Guidi et al. 2003). However, it has been stated that Lepidodasys sp. and Urodasys anorektoxys have stripshaped testes, with no inner lumen or defined sperm ducts (Guidi et al. 2004; Balsamo et al. 2007). The paired testes of Dactylopodola typhle are also strip-shaped, without distinct sperm ducts, but they do have a testicular lumen (Kieneke et al. 2008). The germinal epithelium of the Crasiella species forms the testes wall, like in many species of Macrodasyida (Ruppert 1991). The fusion of the two sperm ducts in a single median, ventral pore is a feature that Crasiella shares with Turbanellidae, Cephalodasys and Megadasys (Remane 1926; Schmidt 1974), whereas most Macrodasyida species have two separate, ventral male pores (Ruppert and Shaw 1977; Ruppert 1978a, b; Guidi et al. 2009; Hummon and Todaro 2010). Only a few species have a direct connection between the sperm duct/s and the caudal organ (Mesodasys, Thaumastodermatidae: Ruppert 1991; Ferraguti and Balsamo 1994; Fregni et al. 1999).

The lack of a sheath enveloping the oocytes is a feature shared by the Crasiella species with other species of Macrodasyida (Mesodasys laticaudatus, Mesodasys adenotubulatus, Paraturbanella teissieri; Ferraguti and Balsamo 1994; Fregni et al. 1999; Balsamo et al. 2002). An incomplete ovarian covering has been reported in some

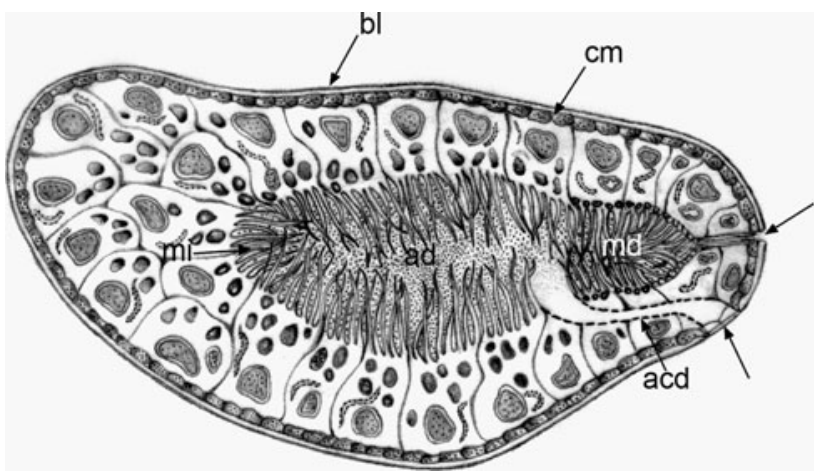

Fig. 6 Schematic drawing of the caudal organ of Crasiella diplura. The basal lamina, the circular musculature and the three ducts of the caudal organ are shown. The accessory duct is on a different focal plane, so it is hatched. The position of the two pores (arrows) of the main and of the accessory duct is clearly visible. acd accessory duct, $a d$ anterior blind duct, bl basal lamina, $\mathrm{cm}$ circular muscles, $m d$ main duct, $m i$ microvilli

species (Dolichodasys carolinensis, Acanthodasys thrinax, Diplodasys ankeli, Tetranchyroderma bunti, Thaumastoderma heideri, Macrodasys sp.; Ruppert and Shaw 1977; Ruppert 1978a, b). A thin epithelial lining the oocytes has been hypothesized to be the plesiomorphic condition in Gastrotricha by Kieneke et al. (2009).

\section{Spermatogenesis}

The occurrence of spermatogenesis in a caudo-cephalic and centripetal direction is typical of most Macrodasyida (Hummon and Hummon 1983; Ruppert 1991; Fischer 1994). The major steps in the process have been documented in five species belonging to five families: Turbanella cornuta, Lepidodasys sp., Cephalodasys maximus, Acanthodasys aculeatus and Urodasys anorektoxys (Teuchert 1976; Fischer 1994; Guidi et al. 2003, 2004; Balsamo et al. 2007). Like in Turbanella cornuta (Turbanellidae), Lepidodasys sp. (Lepidodasyidae) and Cephalodasys maximus, (Cephalodasyidae) the spermatogenesis in species of Crasiella imply a two-step process in the formation of the nuclear-mitochondrial complex. The fusion of mitochondria into a single element is followed by the penetration in the nucleus by this mitochondrial element. A comparable two-step process has also been described in Acanthodasys aculeatus (Thaumastodermatidae), but here the mitochondria are incorporated into the nucleus one by one, and it is only afterwards that they fuse into a single element (Guidi et al. 2003). In Urodasys anorektoxys (Macrodasyidae), spermatogenesis is completely different, because the single mitochondrion gets lost at the end of the process, thus forming a highly specialized sperm that is lacking any mitochondria (Balsamo et al. 2007). In species of Macrodasys, which is the other genus 
of the family, the mitochondrion wraps itself externally around both the nucleus and the acrosome of the mature sperm and remains in this position. Hence, in Macrodasys, the formation of the nuclear-mitochondrial complex likely imply a single step, i.e. the fusion of the mitochondria in a single element (Marotta et al. 2005). This shortened process in species of Macrodasyidae seems to be related to a secondary evolutionary process towards the loss of mitochondria, rather than to a primitive modality of spermatogenesis (Guidi et al. unpublished data).

\section{Sperm}

The spermatozoa of the two Crasiella species resemble those of other macrodasyidan gastrotrichs in several ways.

They have a long and complex acrosome with four different regions: (1) an apical helix with piled discs; (2) a straight tube with helical ridges; (3) a straight tube with radial fibres; and (4) a long and wide region formed by piled vesicles.

The aspects of these regions differ considerably, but three of them have an equivalent in other species. Region one is, in fact, also found in Urodasys anorektoxys (Balsamo et al. 2007), Acanthodasys aculeatus (Guidi et al. 2003), and in the Thaumastodermatinae examined so far; region two is present in Acanthodasys aculeatus, while region four is similar to that observed in Xenodasys eknomios (Guidi et al. 2009) and Thaumastoderma moebjergi (Ferraguti et al., unpublished data). A nucleo-mitochondrial region, formed by a spring-shaped nucleus surrounding the mitochondrion, is also present in all of the Thaumastodermatidae, Lepidodasyidae and Turbanellidae studied so far, as well as in Mesodasys (Ferraguti and Balsamo 1994). It is, however, absent in Xenodasys where, surprisingly, the nuclear spring surrounds part of the acrosome (Guidi et al. 2009). In Crasiella, the flagellum is devoid of a striated cylinder, which is a characteristic present in Thaumastodermatidae, Cephalodasyidae (Hummon and Todaro 2010) and Macrodasyidae, with the exception of Urodasys anorektoxys (Balsamo et al. 2007). The axoneme has a curious modification of the central axonemal apparatus, which is the prominent central sheath that is known only from Clitellata and was regarded as an autapomorphy of that group (Ferraguti 1984). Accordingly, the prominent central sheath of the axoneme is the only characteristic, which clearly separates the spermatozoon of Crasiella from those of the other gastrotrichs.

Accessory sex organs and possible fertilization modality

The two accessory sexual organs of the Crasiella species are always visible, well developed, and close to each other, although they are clearly separate: their morphology and arrangement correspond most closely to those of species of Macrodasys (Macrodasyidae). The shape and size of the frontal organ, as well as the relative position of its external and internal pores, confirm the observations on most Macrodasys species (e.g. Evans 1994).

The structural complexity of the glandulo-muscular caudal organ of Crasiella resembles that of the same organ in the species of Macrodasyidae. In Macrodasys, the posterior part of the caudal organ contains a thin tube, which has a copulatory function (Ruppert 1978a; Evans 1994), while in Urodasys, the organ is V-shaped and formed by two distinct, elongated muscular regions. These are almost parallel to each other, with the left one being glandular in nature, while the right one has a funnel-like, copulatory stylet. The two regions are connected by a thin anterior duct (Schoepfer-Sterrer 1974; Fregni et al. 1998) and open to the outside into a common pore.

The caudal organ is apparently very similar in the species of Crasiella and Macrodasys, because of its shape, and a well-defined, anterior glandular region. However, only in Crasiella, it shows two distinct ducts opening into two distinct pores. The presence of a short, blind duct in the glandular region and of the only circular musculature are two additional features that clearly distinguish the caudal organ of Crasiella from that of the other Macrodasyidae.

A possible mechanism for cross-fertilization in Crasiella is hypothesized here on the basis of morphological grounds and by making a comparison with the few observations available on the fertilization modalities in other Macrodasyida.

A mature individual would first transfer spermatozoa from the testes to the caudal organ. It accomplishes this transfer through ventral body flexions, bringing the male pore close to the pore of the accessory duct of the caudal organ. Spermatozoa enter the accessory duct, and travel to the blind duct of the glandulo-muscular region. They are then stored in the secretion material produced by the surrounding glandular cells (i.e. the abundant electron-dense material that has been observed with TEM) until to be transferred to the mating partner. At mating, the glandulomuscular region pushes spermatozoa from the blind duct through the main duct up to its feed bib-shaped extremity, which might be protruded into the pore of the partner's frontal organ. Active sperm can then reach the mature oocyte via the internal pore of the frontal organ. The supposed fertilization mechanism in Crasiella has some correspondence with that described by Ruppert (1978a) for Macrodasys sp. Here, spermatozoa are transferred during copulation from the male pores of an individual to its own caudal organ through its pore. Inside the caudal organ, they are packed into the thin, copulatory tube at the same time that the latter is placed into the partner's frontal organ. 
Then, the copulatory tube breaks off and releases the spermatozoa, which now head to the mature oocyte. Excess of spermatozoa are reabsorbed.

Some functional similarities and possible homologies between different parts of the reproductive system in the two genera can be suggested on the basis of ultrastructural observations. The lack of a direct connection between the male gonads and the caudal organ in both implies the need to charge the latter with autosperm before fertilization. In fact, the hypothesized mechanisms of fertilization in Macrodasys and Crasiella appear to be similar in that they comprise two subsequent phases, the first for transferring autospermatozoa from the male pore to the caudal organ and the second for transferring sperm to the frontal organ of the partner by means of a specialized anatomical structure belonging to the caudal organ.

According to Ruppert's (1978a) description, the terminal part of the caudal organ of Macrodasys, called 'antrum' by Remane (1936), is a glandular region specialized in receiving autosperm, and thus it may be regarded as being at least functionally correspondent to the accessory lumen of the caudal organ of Crasiella. Similarly, the 'copulatory tube' of Macrodasys (Ruppert 1978a) would have a functional correspondence with the 'feeder bib-shaped' extremity of the caudal organ of Crasiella, since both structures likely transfer autosperm to the partner's frontal organ. However, the timing of the two phases appears to be different for species of Macrodasys and Crasiella, respectively. In the former, the packaging of autosperm into the copulatory tube occurs during copulation, when the tube is already inside the partner's frontal organ; in Crasiella, on the contrary, the autosperm are packaged into the main duct of the caudal organ prior to copulation. All other species of Macrodasyida for which information on reproductive anatomy and biology is available appear to be too different from the Crasiella species to be usefully compared.

\section{Conclusions}

This study has allowed us to trace a consistent plan of the reproductive system in two species of the genus Crasiella. The features of the accessory organs and, in particular, the highly complex structure of the caudal organ suggest that the Crasiella species are phylogenetically close to the Macrodasys species. If the functional similarities that have been proposed for the two genera can be proved to be real homologies, these would strongly support the proximity of the genus Crasiella to the Macrodasyidae, which has been suggested by the phylogenetic analysis of Hochberg and Litvaitis (2001). A morphological study of species of
Planodasys, the only other genus of Planodasyidae, would be the best way to test this hypothesis.

Acknowledgments We would like to thank the referees for their helpful comments. M.A. Todaro is thankful to the organizing committee of the meiofauna workshop at the Sven Lovén Centre for Marine Sciences at Tjärnö, during which Crasiella diplura was collected. The research benefitted from a M.I.U.R. grant to MB (PRIN 2007-Sexuality, reproduction and specific identification of Gastrotricha and Placozoa).

\section{References}

Balsamo M, Ferraguti M, Guidi L, Todaro MA, Tongiorgi P (2002) Reproductive system and spermatozoa of Paraturbanella tessieri (Gastrotricha, Macrodasyida): implications for the sperm transfer modality in Turbanellidae. Zoomorphology 121:235-241

Balsamo M, Guidi L, Pierboni L, Marotta R, Todaro MA, Ferraguti M (2007) Living without mitochondria: spermatozoa and spermatogenesis in two species of Urodasys (Gastrotricha, Macrodasyida) from dysoxic sediments. Invertebr Biol 126:1-9

Balsamo M, d'Hondt JL, Pierboni L, Grilli P (2009) Taxonomic and nomenclatural notes on freshwater Gastrotricha. Zootaxa 2158:1-19

Clausen C (1968) Crasiella diplura gen. et sp. n. (Gastrotricha, Macrodasyoidea). Sarsia 33:59-63

Evans WA (1994) Morphological variability in warm-temperate and subtropical populations of Macrodasys (Gastrotricha: Macrodasyida: Macrodasyidae) with description of seven new species. Proc Biol Soc Washington 107:239-255

Ferraguti M (1984) The comparative ultrastructure of sperm flagella central sheath in Clitellata reveals a new autapomorphy of the group. Zool Scripta 13:201-207

Ferraguti M, Balsamo M (1994) Sperm morphology and anatomy of the genital organs in Mesodasys laticaudatus Remane, 1951 (Gastrotricha, Macrodasyida). J Submicrosc Cytol Pathol 26:21-28

Fischer U (1994) Ultrastructure of spermiogenesis and spermatozoa of Cephalodasys maximus (Gastrotricha, Macrodasyida). Zoomorphology 114:213-225

Fischer U (1996) Ultrastructure of penetrated spermatozoa, ovary, and oogenesis of Dactylopodola baltica (Gastrotricha, Macrodasyida). Invertebr Reprod Dev 29:71-78

Fregni E, Tongiorgi P, Faienza MG (1998) Two new species of Urodasys (Gastrotricha, Macrodasyidae) with cuticular stylet. It J Zool 65:377-380

Fregni E, Balsamo M, Ferraguti M (1999) Morphology of the reproductive system and spermatozoa of Mesodasys adenotubulatus (Gastrotricha, Macrodasyida). Mar Biol 135:515-520

Giere O (2009) Meiobenthology: the microscopic motile fauna of aquatic sediments, 2nd edn. Springer, Berlin

Guidi L, Ferraguti M, Pierboni L, Balsamo M (2003) Spermiogenesis and spermatozoa in Acanthodasys aculeatus (Gastrotricha, Macrodasyida): an ultrastructural study. Acta Zool 84:77-85

Guidi L, Pierboni L, Ferraguti M, Todaro MA, Balsamo M (2004) Spermatology of the genus Lepidodasys Remane, 1926 (Gastrotricha, Macrodasyida): towards a revision of the family Lepidodasyidae Remane, 1927. Acta Zool 85:211-221

Guidi L, Ferraguti M, Todaro MA, Pierboni L, Balsamo M (2009) Unusual spermatozoa and reproductive modalities of Xenodasys eknomios (Gastrotricha: Xenodasyidae). It J Zool 76(2):165-172

Hochberg R, Litvaitis MK (2001) Macrodasyida (Gastrotricha): a cladistic analysis of morphology. Invertebr Biol 120:124-135 
Hummon MR, Hummon WD (1983) Gastrotricha. In: Adiyodi KG, Adiyodi AG (eds) Reproductive biology of invertebrates. Spermatogenesis and sperm function, vol II. Wiley, London, pp 195-205

Hummon WD, Todaro MA (2010) Analytic taxonomy and notes on marine, brackish-water and estuarine Gastrotricha. Zootaxa 2392:1-32

Kieneke A, Arbizu PM, Ahlrichs WH (2008) Anatomy and ultrastructure of the reproductive organs in Dactylopodola typhle (Gastrotricha : Macrodasyida) and their possible functions in sperm transfer. Invertebr Biol 127:12-32

Kieneke A, Ahlrichs WH, Arbizu PM (2009) Morphology and function of reproductive organs in Neodasys chaetonotoideus (Gastrotricha: Neodasys) with a phylogenetic assessment of the reproductive system in Gastrotricha. Zool Scr 38(3):289-311

Marotta R, Guidi L, Pierboni L, Ferraguti M, Todaro MA, Balsamo M (2005) Sperm ultrastructure of Macrodasys caudatus (Gastrotricha: Macrodasyida) and a sperm based phylogenetic analysis of Gastrotricha. Meiofauna Marina 14:9-21

Remane A (1926) Morphologie und Verwandschaftsbeziehungen der aberranten Gastrotrichen I. Z Morph Oecol Tiere 5:625-754

Remane A (1936) Gastrotricha. In: Bronns HG (ed) Klassen Ordnungen des Tierreichs, 4 (2), 1 (2). Gastrotricha und Kinorhyncha. Akad Verlagsgesell, Berlin, pp 1-242

Ruppert EE (1978a) The reproductive system of gastrotrichs. II Insemination in Macrodasys: a unique mode of sperm transfer in Metazoa. Zoomorphologie 89:201-228

Ruppert EE (1978b) The reproductive system of gastrotrichs. III Genital organs of Thaumastodermatinae subfam. n. and
Diplodasyinae subfam. n. with discussion of reproduction in Macrodasyida. Zool Scr 7:93-114

Ruppert EE (1991) Gastrotricha. In: Harrison FW, Ruppert EE (eds) Microscopic anatomy of invertebrates, 4, Aschelminthes. Wiley, New York, pp 41-109

Ruppert EE, Shaw K (1977) The reproductive system of gastrotrichs. I. Introduction with morphological data for two new Dolichodasys species. Zool Scr 6:185-195

Schmidt P (1974) Interstitielle fauna von Galapagos. IV. Gastrotricha. Mikrofauna des Meeresbodens 26:1-76

Schoepfer-Sterrer C (1974) Five new species of Urodasys and remarks on the terminology of the genital organs in Macrodasyida (Gastrotricha). Cah Biol Mar 15:229-259

Teuchert G (1968) Zur Forpflanzung und Entwicklung der Macrodasyoidea (Gastrotricha). Z Morph Ökol Tiere 63:343-418

Teuchert G (1976) Elektronenmikroskopische Untersuchung über die Spermatogenese und Spermatohistogenese von Turbanella cornuta Remane (Gastrotricha). J Ultrastruct Res 56:1-14

Thane-Fenchel A (1970) Interstitial gastrotrichs in some south Florida beaches. Ophelia 7:113-137

Willems WR, Curini-Galletti M, Ferrero TJ, Fontaneto D, Heiner I, Huys R, Ivanenko VN, Kristensen RM, Kanneby T, MacNaughton MO, Martínez Arbizu P, Todaro MA, Sterrer W, Jondelius U (2009) Meiofauna of the Koster-area, results from a workshop at the Sven Lovén Centre for Marine Sciences (Tjärnö, Sweden). Meiofauna Marina 17:1-34 